\title{
Factores protectores contra la automedicación en personas con enfermedad reciente que no buscaron atención en un establecimiento de salud
}

\section{Protective factors against self-medication among people with a recent illness who did not seek for attention at a healthcare facility}

\author{
Manuel André Virú-Loza ${ }^{1,2}$ \\ ${ }^{1}$ Médico residente de Pediatría de la Facultad de Medicina de San Fernando, Universidad Nacional Mayor de San Marcos. \\ ${ }^{2}$ Asociación para el Desarrollo de la Investigación Estudiantil en Ciencias de la Salud (ADIECS-UNMSM).
}

\begin{abstract}
Correspondencia
Manuel Virú-Loza

Direcc. Jr. Piedra Bigua 2241-A, La Huayrona, San Juan de Lurigancho. m.andre.viru@gmail.com
\end{abstract}

Recibido: 2 de abril 2017.

Aceptado: 12 de agosto 2017.

Conflictos de interés: el autor declara no tener conflictos de interés.

Financiamiento: autofinanciado.

Citar como: Virú-Loza MA. Factores protectores contra la automedicación en personas con enfermedad reciente que no buscaron atención en un establecimiento de salud. An Fac med. 2017;78(4):398-404 DOI: http://dx.doi.org/10.15381/ anales.v78i4.14260

\section{An Fac med. 2017;78(4):398-404 / http://dx.doi.org/10.15381/anales.v78i4.14260}

Resumen

Introducción: En Perú faltan estudios publicados sobre automedicación que hayan sido realizados a nivel nacional. La mayoria de la información disponible es descriptiva y de sectores pequeños de la población. Objetivos: El objetivo del presente estudio fue analizar si factores relacionados a la accesibilidad a los servicios de salud, a la percepción sobre la enfermedad, los médicos y la medicina tradicional, así como también factores socioeconómicos, están asociados con la automedicación en personas con una enfermedad reciente que no buscaron atención en un establecimiento de salud. Diseño: Análisis transversal a partir de la Encuesta Nacional de Hogares (ENAHO) del año 2015. Lugar: Estudio nacional. Sujetos de estudio: Aquellos que tuvieron una enfermedad en las últimas 4 semanas y no buscaron atención en un establecimiento de salud. Intervenciones: Se calculó medidas de resumen. Se utilizó regresión logística bivariada y multivariada. Resultados: Todas las variables de interés resultaron factores protectores significativos tanto en el análisis bivariado como multivariado. Conclusiones: Las variables de interés fueron factores protectores, confiriendo mayor protección el hecho de no tener dinero.

Palabras clave: Automedicación; Accesibilidad a los servicios de salud; Percepción; Medicina tradicional; Factores socioeconómicos.

\section{Abstract}

Introduction: In Peru there is a lack of published studies about self-medication that had been performed nationwide. Most of the information available is descriptive and from small sectors of the population. Objectives: The aim of this study was to analyze if factors related with health services accessibility, perception about disease, doctors and traditional medicine, as well as socioeconomic factors, are associated with selfmedication among people with a recent illness who did not seek for attention at a healthcare facility. Design: Transversal analysis from the National Household Survey (ENAHO) of year 2015. Setting: Nationwide study. Study subjects: Those who had an illness in the last 4 weeks and did not look for attention in a healthcare facility. Interventions: Summary measures were calculated. Bivariate and multivariate logistic regression were used. Results: All variables of interest resulted significant protective factors in both bivariate and multivariate analysis. Conclusions: Variables of interest were significant protective factors, conferring more protection the fact of not having money.

Keywords: Self medication; Health services accessibility; Perception; Traditional medicine; Socioeconomic factors. 


\section{INTRODUCCIÓN}

La prevalencia de la automedicación en el mundo es muy variable y es casi imposible medir su prevalencia con precisión como ya han mencionado otros autores ${ }^{(1)}$. Actualmente existen muchos estudios publicados sobre automedicación; sin embargo algunos investigadores afirman que no es suficiente ya que la mayoría de ellos sólo describen el problema pero parece ser que la discusión acerca de este tema en muchos casos no llega a un análisis detallado ${ }^{(1)}$.

De acuerdo a la Organización Mundial de la Salud (OMS), la automedicación se define como la selección y uso de medicamentos para tratar síntomas y enfermedades autoreportadas sin el consejo de un profesional de la salud calificado para una cierta función. Además involucra el uso de productos medicinales por el consumidor para tratar desórdenes o síntomas autoreconocidos, o el uso intermitente o continuo de medicación prescrita por un médico para enfermedades o síntomas crónicos o recurrentes. También menciona que en la práctica, también incluye el uso de medicación de miembros de la familia, especialmente cuando está involucrado el tratamiento de niños o ancianos ${ }^{(2,3)}$.

La automedicación podría ser considerada un problema debido a los riesgos a los que conlleva; sin embargo, también constituye una oportunidad ya que mucha gente tiene dificultades para acceder a establecimientos de salud. En algunos sitios, más del $70 \%$ de las personas que se automedican admiten no tener ningún conocimiento acerca de la medicación que piden ${ }^{(4)}$, motivo que incrementa el riesgo que representa la automedicación y hace importante investigar sus causas. Conocer las razones que utiliza la gente para automedicarse puede mejorar la perspectiva que se tiene sobre cómo plantear soluciones o identificar oportunidades.

En la Ley General de Salud de Perú, las medicinas están clasificadas de acuerdo a las condiciones en las que pueden ser vendidas: sólo con prescripción especial, prescripción médica y provista sólo por farmacias; sin prescripción mé- dica y provista sólo por farmacias; y sin prescripción médica pero provista no exclusivamente por establecimientos farmacéuticos. Al mismo tiempo, la ley también establece que el farmacéutico es responsable de la dispensación, información y orientación del usuario acerca del uso de medicamentos; pero no está autorizado para prescribir. Desde 2004 la Dirección General de Medicamentos, Insumos y Drogas (DIGEMID) del Ministerio de Salud del Perú ha realizado actividades informativas y ha diseñado un manual sobre la enseñanza del uso racional de medicamentos que ha sido renovado en el 2012, sin embargo las medidas de impacto de estas estrategias no han sido publicadas. Como puede observarse, existe una posición sobre la necesidad de informar a la población sobre el uso racional de medicamentos, lo cual tiene que incluir mejorar el rol informativo del farmacéutico ${ }^{(5)}$.

La influencia que poseen las farmacias sobre la automedicación en otros países ${ }^{(6,7)}$ y en Perú ${ }^{(8,9)}$ está descrita. Incluso en otros países las farmacias fueron identificadas como críticas para asegurar el acceso a servicios de atención primaria en salud ${ }^{(10,11)}$ y un lugar ideal para la promoción en salud ${ }^{(12)}$. Sin embargo, existen muchos otros factores que también influyen en la automedicación, y estos requieren entendimiento y atención por parte de las instancias de salud en el Perú.

En Perú faltan estudios publicados que hayan sido realizados a nivel nacional. La mayoría de la información disponible es descriptiva y de sectores pequeños de la población. Existe la necesidad de generar más discusión acerca de la automedicación y sus causas en el Perú. Así, el objetivo del presente estudio fue determinar los factores asociados a automedicación en personas con una enfermedad reciente que no buscaron atención en un establecimiento de salud, investigando los factores relacionados a posibles razones para no buscar este tipo de atención.

\section{MÉTODOS}

Se realizó un estudio observacional transversal. Todos los datos analizados provienen del Módulo de Salud de la Encuesta
Nacional de Hogares (ENAHO), disponible libremente en el sitio web del Instituto Nacional de Estadística e Informática (INEI) de Perú (13) Se utilizó información de la base de datos de la encuesta anual del 2015.

Primero, los entrevistados que no reportaron tener algún signo o síntoma (como tos, cefalea, fiebre, nauseas), enfermedad aguda (como resfrío, colitis, etc.), o recaída de una enfermedad crónica o accidente en las últimas 4 semanas fueron excluidos del estudio. Cabe resaltar que no se registró información detallada de cuál fue el cuadro clínico o diagnóstico de cada persona, ni tampoco hubo evaluación médica de las personas encuestadas, ya que esta información no estuvo contemplada en la metodología de la ENAHO ${ }^{(13)}$. En segundo lugar, los entrevistados que buscaron atención en un establecimiento de salud debido a cualquiera de los problemas médicos previamente descritos también fueron excluidos del estudio. De esta manera los únicos seleccionados para el estudio fueron aquellos que tuvieron una enfermedad reciente y no buscaron atención en un establecimiento de salud.

En el ENAHO, existe una pregunta que se formula de la siguiente manera: “¿Cuáles son las razones por las que no acudió a un centro o establecimiento de salud?" Esta pregunta ofrece al encuestado múltiples opciones de respuesta para escoger, entre las cuales figuran: (a) "no tuvo dinero"; (b) "se encuentra lejos"; (c) "demoran mucho en atender"; (d) "no confía en los médicos"; (e) "no era grave / no fue necesario"; (f) "prefiere curarse con remedios caseros"; (g) "no tiene seguro"; (h) "se auto recetó o repitió receta anterior"; (i) "falta de tiempo"; (j) "por el maltrato del personal de salud" y (k) "otro (especificar)" (13). Se definió "automedicación" en el presente estudio como el hecho de escoger la opción "se auto recetó o repitió receta anterior" al responder la pregunta mencionada. El resto de opciones de respuesta fueron consideradas las variables de interés potencialmente asociadas a la automedicación. Cabe resaltar que las categorías de respuesta a la mencionada pregunta no son mutuamente excluyentes (la encuesta menciona de forma explícita que 
acepta una o más alternativas en esta pregunta). Se consideraron como potenciales variables intervinientes a la edad, el género y el nivel educativo.

Para analizar los datos, se utilizó el software STATA v.12. Se realizó un análisis descriptivo de las variables sociodemográficas (edad, género, nivel educativo, número de viviendas en el área y área geográfica) y de las variables de interés. Se realizaron análisis bivariados de las variables de interés y de las potenciales intervinientes, siendo todos ellos análisis de regresión logística. Finalmente, se realizó un análisis de regresión logística multivariada sólo con las variables de interés (9 variables: "no tener suficiente dinero", "se encuentra lejos", "demoran mucho en atender", "no confía en los médicos", "no era grave / necesario", "prefiere curarse con remedios caseros", "no tiene seguro", "falta de tiempo", y "maltrato del personal de salud"). Se calcularon los OR para cada variable en todos los análisis de regresión realizados. Además, para realizar los análisis mencionados, los datos fueron ponderados con el factor de expansión que viene incluido en la base de datos con la finalidad de ser utilizado en los análisis que se realicen con los datos de dicha encuesta.

\section{RESULTADOS}

Se estudiaron 45261 personas, de las cuales el $21 \%$ refirieron que su preferencia para automedicarse fue la razón para no buscar atención en un establecimiento de salud. Entre estos sujetos, la mayoría de ellos tenían educación primeria incompleta $(19,2 \%)$, vivían en zonas con 20001 a 100000 viviendas (24,5\%) y vivían en la selva $(26,4 \%)$ (Tabla 1$)$.

La edad (OR: 1,000; IC95\%: 1,0011,004) y el género (OR: 1,08; IC95\%: $1,02-1,15)$ fueron factores de riesgo estadísticamente significativos; sin embargo, el tamaño del efecto que tienen es insignificante. El nivel educativo fue analizado por etapas terminadas, de las cuales haber completado la educación primaria (OR: 1,15; IC95\%: 1,07 - 1,24), secundaria (OR: 1,28; IC95\%: 1,19-1,37) y superior (OR: 1,50; IC95\%: 1,35 - 1,66) fueron factores de riesgo estadísticamen- te significativos. Tener posgrado no fue un factor con asociación estadísticamente significativa (OR: 1,42; IC95\%: 0,982,06) (Tabla 1).

En el análisis bivariado de potenciales factores asociados, no tener dinero (OR: 0,25; IC95\%: 0,21-0,30), referir que el centro de salud se encuentra lejos (OR: 0,34; IC95\%: 0,28 - 0,42), referir que demoran mucho en atender (OR: 0,46; IC95\%: 0,39-0,54), no confiar en los médicos (OR: 0,29; IC95\%: 0,23 - 0,36), la percepción de que el problema de salud no era grave / no era necesario acudir a un centro de salud (OR: 0,33; IC95\%: 0,30 - 0,36), preferir remedios caseros (OR: 0,32; IC95\%: 0,28-0,36), no tener seguro (OR: 0,37; IC95\%: 0,27-0,50), falta de tiempo (OR: 0,28; IC95\%: 0,25 - 0,32) y maltrato del personal de salud (OR: 0,27; IC95\%: 0,19-0,38), fueron factores protectores estadísticamente significativos (Tabla 1).

En el análisis multivariado las variables de interés resultaron tener también un efecto protector estadísticamente significativo. La variable que confirió la mayor protección fue no tener suficiente dinero (OR ajustado: 0,042; IC95\%: 0,03-0,05) (Tabla 2).

\section{DISCUSIÓN}

En el caso del nivel educativo, se trata de variables complejas sobre las que no se garantiza la homogeneidad de la calidad educativa de toda la población en los distintos niveles, haciendo por tanto algo superficial la interpretación de dichos resultados. En vista de esta dificultad se podría realizar un análisis que incluya otras variables que puedan ser de utilidad para al menos aproximarse a poder estudiar esta potencial heterogeneidad, sin embargo esto escapa a los fines de este estudio. En el caso de la educación superior, además de lo ya mencionado, amerita otros análisis que tengan en cuenta la diferenciación entre educación superior universitaria y no universitaria. Por estos motivos, se decidió no incluir al nivel educativo en el análisis multivariado, ya que haría poco precisa la interpretación de los resultados en dicho análisis y sería más prudente su análisis detallado en estudios realizados específicamente para responder estas interrogantes. Sin embargo, sí se consideró relevante mostrar los hallazgos del análisis bivariado de esta variable. En el caso de las variables edad y género, el tamaño del efecto de estas variables en el análisis bivariado no es significativo por lo que tampoco fueron incluidas en el análisis multivariado.

En población China, se ha descrito mayor proporción de automedicación entre aquellos que vivían en pobreza en 1998 y 2008, pero lo inverso se encontró en el $2003^{\text {(14); }}$ por otro lado, un estudio en el mismo país en el año 2013 evidenció que el costo de la atención hospitalaria fue la tercera razón principal para preferir la automedicación ${ }^{(15)}$. De forma similar, en Colombia la falta de dinero fue identificada como la principal causa de automedicación ${ }^{(16)}$. De la misma manera, en un estudio en adultos mayores en Perú, en un $37 \%$ de sujetos la falta de dinero fue una razón para automedicarse ${ }^{(17)}$. Contrario a todo lo expuesto, en un estudio en adolescentes de Brasil no hubo diferencia entre quintiles de posición socioeconómica con respecto a la automedicación ${ }^{(18)}$.

En el presente estudio, no tener dinero tuvo una influencia negativa en la automedicación. Una posible explicación es que la población analizada puede tener demasiadas limitaciones socioeconómicas y por tanto si perciben que su problema no es severo preferirían no consumir medicamentos (lo cual incluye a la automedicación) para ahorrar dinero; por otro lado, si perciben que su problema de salud necesita tratamiento algunos preferirían tomar remedios caseros en lugar de posiblemente gastar más dinero en medicinas.

Efectos nulos o positivos en la automedicación en otros estudios pueden deberse a los grupos de edad estudiados como en el caso del estudio en adolescentes de Brasil (18) o a posibles cambios de contexto social o económico que puedan hacer variar esta relación con el tiempo como lo observado en el estudio realizado con datos de tres años diferentes en China ${ }^{(14)}$. Esta asociación merece más análisis en futuros estudios. De forma similar, el hecho de que el no contar con seguro médico haya resultado ser 
Tabla 1. Análisis descriptivo y bivariado de regresión logística de las variables estudiadas.

\begin{tabular}{|c|c|c|c|c|}
\hline & \multicolumn{2}{|c|}{ Automedicado } & \multicolumn{2}{|c|}{ Análisis bivariado } \\
\hline & Sí (9531 sujetos) & No (35730 sujetos) & $\mathrm{OR}$ & $95 \%$ IC \\
\hline Edad (años) & $36,1 \pm 22,9^{*}$ & $35,4 \pm 21,8^{*}$ & 1,000 & 1,001 a 1,004 \\
\hline Género (masculino) & $4383(45,9 \%)$ & $17090(47,8 \%)$ & 1,08 & 1,02 a 1,15 \\
\hline Nivel educativo & & & --- & --- \\
\hline Sin educación & $723(7,5 \%)$ & $3298(9,2 \%)$ & --- & --- \\
\hline Educación pre-escolar & $425(4,4 \%)$ & $1272(3,5 \%)$ & --- & --- \\
\hline Educación primaria incompleta & $1833(19,2 \%)$ & $7737(21,6 \%)$ & --- & --- \\
\hline Educación primaria completa & $1110(11,6 \%)$ & $4448(12,4 \%)$ & 1,15 & 1,07 a 1,24 \\
\hline Educación secundaria incompleta & $1419(14,8 \%)$ & $5823(16,3 \%)$ & --- & --- \\
\hline Educación secundaria completa & $1695(17,7 \%)$ & $6283(17,5 \%)$ & 1,28 & 1,19 a 1,37 \\
\hline Educación superior incompleta & $830(8,7 \%)$ & $2656(7,4 \%)$ & --- & --- \\
\hline Educación superior completa & $1090(11,4 \%)$ & $2965(8,3 \%)$ & 1,50 & 1,35 a 1,66 \\
\hline Post-grado & $88(0,9 \%)$ & $228(0,6 \%)$ & 1,42 & 0,98 a 2,06 \\
\hline Viviendas en el área & & & --- & --- \\
\hline Más de 100000 viviendas & $1450(15,2 \%)$ & $4320(12,0 \%)$ & --- & --- \\
\hline 20001 a 100000 viviendas & $2336(24,5 \%)$ & $6388(17,8 \%)$ & --- & --- \\
\hline 10001 a 20000 viviendas & $714(7,4 \%)$ & $2713(7,5 \%)$ & --- & --- \\
\hline 4001 a 10000 viviendas & $669(7,0 \%)$ & $2452(6,8 \%)$ & --- & -- \\
\hline 401 a 4000 viviendas & $1296(13,6 \%)$ & $4793(13,4 \%)$ & --- & --- \\
\hline Menos de 401 viviendas & $351(3,6 \%)$ & $1612(4,5 \%)$ & --- & --- \\
\hline Área de empadronamiento rural compuesta & $2064(21,6 \%)$ & $10129(28,3 \%)$ & --- & --- \\
\hline Área de empadronamiento rural simple & $651(6,8 \%)$ & $3323(9,3 \%)$ & --- & -- \\
\hline Área geográfica & & & --- & --- \\
\hline Costa norte & $1040(10,9 \%)$ & $4444(12,4 \%)$ & --- & --- \\
\hline Costa central & $1531(16,0 \%)$ & $2594(7,2 \%)$ & --- & --- \\
\hline Costa sur & $344(3,6 \%)$ & $1892(5,3 \%)$ & --- & --- \\
\hline Sierra norte & $438(4,6 \%)$ & $2435(6,8 \%)$ & --- & --- \\
\hline Sierra central & $1657(17,3 \%)$ & $7480(20,9 \%)$ & --- & --- \\
\hline Sierra sur & $843(8,8 \%)$ & $6223(17,4 \%)$ & --- & --- \\
\hline Selva & $2522(26,4 \%)$ & $7656(21,4 \%)$ & --- & --- \\
\hline Lima Metropolitana & $1156(12,1 \%)$ & $3006(8,4 \%)$ & --- & --- \\
\hline Razones para no buscar atención en un establecimiento de salud & & & --- & --- \\
\hline Afirmar no tener suficiente dinero & $265(2,7 \%)$ & $2928(8,1 \%)$ & 0,25 & 0,21 a 0,30 \\
\hline Afirmar que se encuentra lejos el establecimiento de salud & $314(3,2 \%)$ & $2471(6,9 \%)$ & 0,34 & 0,28 a 0,42 \\
\hline Afirmar que demoran mucho en atender en el establecimiento de salud & $490(5,1)$ & $3994(11,1)$ & 0,46 & 0,39 a 0,54 \\
\hline Afirmar que no confía en los médicos & $184(1,9 \%)$ & $2059(5,7 \%)$ & 0,29 & 0,23 a 0,36 \\
\hline $\begin{array}{l}\text { Afirmar que padecimiento no era grave / no era necesario ir al } \\
\text { establecimiento de salud }\end{array}$ & $2583(27,1 \%)$ & $17607(49,2 \%)$ & 0,33 & 0,30 a 0,36 \\
\hline Afirmar que prefiere remedios caseros & $740(7,7 \%)$ & $7125(19,9 \%)$ & 0,32 & 0,28 a 0,36 \\
\hline Afirmar que no tiene seguro & $105(1,1 \%)$ & $919(2,5 \%)$ & 0,37 & 0,27 a 0,50 \\
\hline Afirmar que le falta de tiempo & $623(6,5 \%)$ & $6361(17,8 \%)$ & 0,28 & 0,25 a 0,32 \\
\hline Afirmar que existe maltrato del personal de salud & $64(0,6 \%)$ & $671(1,8 \%)$ & 0,27 & 0,19 a 0,38 \\
\hline Otros & $155(1,6 \%)$ & $1536(4,3 \%)$ & --- & --- \\
\hline
\end{tabular}

*Media \pm desviación estándar. 
Tabla 2. Análisis de regresión logística multivariada.

\begin{tabular}{|c|c|}
\hline Razones para no buscar atención en un establecimiento de salud & OR (IC95\%) \\
\hline Afirmar no tener suficiente dinero & $0,042(0,03$ a 0,05$)$ \\
\hline Afirmar que se encuentra lejos el establecimiento de salud & $0,07(0,05$ a 0,09$)$ \\
\hline Afirmar que demoran mucho en atender en el establecimiento de salud & $0,08(0,07$ a 0,10$)$ \\
\hline Afirmar que no confía en los médicos & $0,05(0,03$ a 0,06$)$ \\
\hline Afirmar que padecimiento no era grave / no era necesario ir al establecimiento de salud & $0,05(0,04$ a 0,05$)$ \\
\hline Afirmar que prefiere remedios caseros & $0,06(0,05$ a 0,07$)$ \\
\hline Afirmar que no tiene seguro & $0,07(0,04$ a 0,10$)$ \\
\hline Afirmar que le falta de tiempo & $0,047(0,03$ a 0,05$)$ \\
\hline Afirmar que existe maltrato del personal de salud & $0,06(0,04$ a 0,09$)$ \\
\hline
\end{tabular}

también un factor protector podría estar reflejando carencias económicas para acceder a pagar un seguro médico, y que por tanto este resultado no haga más que estar reflejando el efecto protector de referir no tener suficiente dinero. Sin embargo, ante la existencia de seguros de salud gratuitos como el Seguro Integral de Salud del Ministerio de Salud y de otras circunstancias en las que un tercero esté solventando el seguro del encuestado, también sería necesario realizar otro estudio para entender mejor esta asociación.

El referir que el centro de salud se encuentra lejos, que demoran mucho en atender y que falta de tiempo, son aspectos diferentes que pueden de cierta forma relacionarse a problemas de acceso a los servicios de salud, ya reportados como causas de automedicación en otros estudios ${ }^{(19,20,21,22)}$. Se podría inferir que a mayor dificultad para el acceso hay mayor tendencia a preferir la automedicación como en otros estudios $(16,23,24,25)$. Sin embargo, en el presente estudio son factores protectores. Una posible explicación sería que en este grupo de sujetos al mismo tiempo haya una desidia por recibir alguna medicación, para lo cual tendrían que posiblemente estar pasando por problemas de salud que consideran que no son severos y que "pueden esperar". Esta hipótesis análoga a la planteada para explicar por qué el no tener suficiente dinero es un factor protector, también queda pendiente de ser aclarada en futuras investigaciones.
Los encuestados que refieren maltrato del personal de salud como motivo para automedicarse estarían en un contexto similar a aquellos que refieren que se automedican debido a que no confían en los médicos. Ambos comparten percepciones negativas hacia el personal de salud. En otros estudios este tipo de percepciones negativas - maltrato del personal de salud ${ }^{(15)}$, desconfianza en los médicos ${ }^{(23,24,25,26)}$ y opinión negativa sobre todo el sistema de salud ${ }^{(27)}$ - se describen como causas de automedicación. En un estudio realizado en Colombia se describió como motivos para no asistir a ver al médico al hecho de no confiar en el médico y al hecho no confiar en los medicamentos; y además encontraron que más del $60 \%$ de las personas que no asistían al médico afirmaban recurrir a hierbas, remedios caseros, homeopatía y otras terapias alternativas (16). Podría significar que al menos un grupo de sujetos con percepciones negativas hacia la medicina convencional (médicos y medicamentos convencionales) responden eligiendo utilizar remedios caseros o alternativos. En el análisis realizado en el presente estudio, utilizar remedios caseros disminuye el riesgo de automedicación, abriendo la posibilidad de que esto se deba al menos en parte a la hipótesis expuesta. Si esto fuera así en nuestra población, tendría que encontrarse asociaciones positivas entre estas percepciones negativas y el uso de remedios caseros, y a su vez una asociación positiva entre las percepciones negativas hacia los médicos y los medicamentos por separado. Estas y otras hipótesis relacionadas a la reacción de la gente hacia los médicos y medicamentos convencionales, y cómo esto influye en la automedicación merece un estudio aparte.

Con respecto a la percepción de que el problema de salud "no es grave" o que "no es necesario acudir a un centro de salud" y su efecto en disminuir el riesgo de automedicación, en Hong Kong se reportó que la autopercepción de una "pobre salud" favoreció la automedicación en adolescentes ${ }^{(28)}$. De forma similar, en adolescentes de Brasil se observó que la prevalencia del uso de medicamentos fue mayor a la esperada entre aquellos que autocalificaron su salud como "pobre o muy pobre" (18). Ambos coinciden con el análisis del presente estudio. Por el contrario, en Latinoamérica hay varios reportes de la autopercepción del problema de salud como no severo como causa de automedicación (20,21,29,30). Además, un estudio en Jordania sugirió a la percepción de dolencias como leves como una razón potencial para preferir la automedicación ${ }^{(24)}$ y en Irán un meta-análisis demostró esta asociación en la misma dirección (25) al igual que un estudio en China ${ }^{(15)}$. De la misma manera, en España se encontró que una autopercepción del estado de salud positiva estuvo asociada a una mayor probabilidad de automedicarse ${ }^{(31)}$. En el presente estudio, se coincidiría con los dos estudios que muestran que una percepción de salud pobre favorece la automedicación. Sin embargo, los demás muestran que una percepción de que el problema de salud no es grave es la que favorece la automedicación. Es necesario aclarar todos los aspectos que involucran este tipo de percepción para entender mejor esta asociación.

En un estudio en realizado en China, la segunda alternativa más común entre los que se automedicaron fue la medicina tradicional ${ }^{(15)}$. En adultos españoles el uso de la medicina tradicional estuvo asociado con una mayor probabilidad de automedicación ${ }^{(31)}$, de forma similar en México se describió un grupo poblacional con gran predisposición a la medicina alternativa y la automedicación ${ }^{(32)}$. No se tiene claro si el término "medicina tradicional" o "medicina alternativa" en otros estudios necesariamente estaría hacien- 
do alusión a lo que los encuestados entendieron como "remedios caseros". Al no haber mucha información respecto a esta asociación, sería importante realizar un estudio que muestre si en nuestro contexto el rol protector contra la automedicación del uso de remedios caseros se debe a un factor económico de fondo, al hecho de desconfiar en la medicina convencional de acuerdo a la hipótesis expuesta previamente, o en última instancia a un problema conceptual al existir falta de uniformidad entre las definiciones de este tipo de terapias "no convencionales" entre los diferentes estudios.

La primera limitación de este estudio es que utiliza una base de datos secundaria. También existe limitación al comparar resultados con otros estudios ya que no hay una única definición de automedicación entre los mismos ${ }^{(33)}$. Por otro lado el hecho de que la ENAHO no cuente con información detallada sobre el cuadro clínico o diagnóstico de los pacientes ${ }^{(13)}$, así como el hecho de que los mismos no hayan recibido evaluación médica como parte de la metodología de la encuesta también debe tenerse en cuenta al momento de interpretar los resultados. Sin evaluación médica no se puede definir el diagnóstico de cada persona; y esto nos imposibilita de analizar las relaciones existentes entre la percepción de la persona sobre su dolencia, su diagnóstico real y su tendencia a la automedicación. Sin embargo, esto abre posibilidad a nuevos estudios que profundicen en este punto. Por otro lado, la definición de automedicación en el presente estudio se basa en la información brindada por la ENAHO, no en las definiciones brindadas por instituciones u organismos afines a la problemática. A futuro se recomienda realizar estudios a nivel nacional utilizando la definición de la OMS. No se informa sobre quienes incurrieron en automedicación responsable debido a que esta información no está disponible en la base de datos estudiada, lo cual es un sesgo a tener en cuenta. No se analizó más variables relacionadas a la condición socioeconómica de los encuestados debido a que este tipo de preguntas no se encuentran en el Módulo de Salud de la ENAHO, y debido a su complejidad queda pendiente para un próximo estudio.
Finalmente, la ENAHO no fue diseñada de forma específica para realizar estudios sobre automedicación ni para estimar su prevalencia.

Como conclusión, las variables de interés analizadas resultaron ser factores protectores contra la automedicación, principalmente el hecho de referir no tener dinero. Quedan muchas preguntas relacionadas a entender con más detalle cada una de estas asociaciones complejas.

\section{REFERENCIAS BIBLIOGRÁFICAS}

1. Zhao Y, Ma S. Observations on the prevalence, characteristics, and effects of self-treatment. Front Public Health. 2016;4:69. DOI: 10.3389/ fpubh.2016.00069

2. World Health Organization. The role of the pharmacist in self-care and self-medication [Internet]. Geneva: World Health Organization; 1998 [Fecha de acceso: 10 de enero de 2017]. Disponible en: http://apps.who.int/medicinedocs/pdf/whozip32e/ whozip32e.pdf

3. World Health Organization. Guidelines for the regulatory assessment of medicinal products for use in self-medication [Internet]. Geneva: World Health Organization; 2000 [Fecha de acceso: 10 de enero de 2017]. Disponible en: http://apps.who. int/medicinedocs/pdf/s2218e/s2218e.pdf

4. Fuentes Albarrán K, Villa Zapata L. Analysis and quantification of self-medication patterns of customers in community pharmacies in southern Chile. Pharm World Sci. 2008;30:863-868. DOI: 10.1007/ s11096-008-9241-4

5. Vásquez S. Automedicación en el Perú (Internet). Lima: Dirección General de Medicamentos, Insumos y Drogas (DIGEMID); 2008 (citado el 8 de octubre de 2016). Disponible en: http://www. digemid.minsa.gob.pe/upload/uploaded/pdf/ automedicacion_junio_2008.pdf

6. Wazaify M, Al-Bsoul-Younes A, Abu-Gharbieh E, Tahaineh L. Societal perspectives on the role of community pharmacists and over-the-counter drugs in Jordan. Pharm World Sci. 2008;30(6):88491. DOI: 10.1007/s11096-008-9244-1

7. Brata C, Gudka S, Schneider CR, Clifford RM. A review of the provision of appropriate advice by pharmacy staff for self-medication in developing countries. Res Social Adm Pharm. 2015;11(2):13653. DOI: 10.1016/j.sapharm.2014.07.003

8. Hermoza-Moquillaza R, Loza-Munarriz C, Rodríguez-Hurtado D, Arellano-Sacramento C Hermoza-Moquillaza V. Automedicación en un distrito de Lima metropolitana, Perú. Rev Med Hered. 2016;27:15-21. DOI: http://dx.doi.org/10.20453/ rmh.v27i1.2779

9. Ecker L, Ruiz J, Vargas M, Del Valle LJ, Ochoa TJ. Prevalencia de compra sin receta y recomendación de antibióticos para niños menores de 5 años en farmacias privadas de zonas periurbanas en Lima, Perú. Rev Peru Med Exp Salud Publica. 2016;33(2):215-23. DOI: http://dx.doi. org/10.17843/rpmesp.2016.332.2152

10. Fang Y, Yang S, Zhou S, Jiang M, Liu J. Community pharmacy practice in China: past, present and future. Int J Clin Pharm.2013;35:520-528. DOI: 10.1007/s11096-013-9789-5

11. Wong FY, Chan FW, You JH, Wong EL, Yeoh EK. Patient self-management and pharmacist-led patient self-management in Hong Kong: A focus group study from different healthcare professionals' perspectives. BMC Health Serv Res. 2011;11:121. DOI: 10.1186/1472-6963-11-121

12. Boardman $H$, Lewis $M$, Croft $P$, Trinder $P$, Rajaratnam $G$. Use of community pharmacies: a population-based survey. J Public Health (Oxf). 2005;27(3):254-62. DOI: 10.1093/pubmed/fdi032

13. Microdatos [base de datos en internet]. Perú: Instituto Nacional de Estadistica e Informática [fecha de acceso: 15 de enero de 2017]. Disponible en: http://iinei.inei.gob.pe/microdatos/

14. Yuefeng L, Keqin R, Xiaowei R. Use of and factors associated with self-treatment in China. BMC Public Health. 2012;12:995. DOI: 10.1186/14712458-12-995

15. Jiang $Y$, Wang $Y$, Li Y, Wang X, Ma C, Ma S. Prevalence, characteristics, and cost of self-treatment in the middle-aged and elderly: observations from Henan, China. Public Health. 2015;129(5):597-600. DOI: 10.1016/j.puhe.2015.02.005

16. López JJ, Dennis R, Moscoso SM. Estudio sobre la automedicación en una localidad de Bogotá. Rev salud pública. 2009;11(3):432-442. DOI: https://doi. org/10.1590/S0124-00642009000300012

17. Mini EH, Rojas LF, Balabarca CY, Espinoza P, Marin HG, Quispe FJ, et al. Factores relacionados con la automedicación en adultos mayores. An Fac med. 2010;71 Supl 1

18. Bertoldi AD, Camargo AL, Silveira MP, Menezes AM, Assunção MC, Gonçalves H, et al. Selfmedication among adolescents aged 18 years: the 1993 Pelotas (Brazil) birth cohort study. J Adolesc Health. 2014;55(2):175-81. DOI: 10.1016/j. jadohealth.2014.02.010

19. Mini E, Varas R, Vicuña $Y$, Lévano M, Rojas L, Medina $\mathrm{J}$, et al. Automedicación en gestantes que acuden al instituto nacional materno perinatal, Perú 2011. Rev Peru Med Exp Salud Publica. 2012; 29(2):212-17

20. Chavez D, Castro J. Automedicación en zonas urbana y urbano-marginal en la provincial de Lima (Perú). Rev Med Carrionica. 2017; 4(1):22-35

21. Fajardo-Zapata A, Méndez-Casallas $F$, HernándezNiño J, Molina L, Milena A, Nossa C, et al. La automedicación de antibióticos: un problema de salud pública. Salud Uninorte. Barranquilla (Col.). 2013;29(2):226-235

22. Villegas F, Nasner K, Buitrago D, Cruz A, Ruden $S$, Bedoya J, et al. Patrón de automedicación en la zona urbana de la ciudad de Pereira (Colombia) en el trimestre marzo-mayo 2013. Rev Ingestigaciones Andina. 2014;16(29):1073-85

23. Shaghaghi A, Asadi M, Allahverdipour H. Predictors of self-medication behavior: a systematic review. Iran J Public Health. 2014;43(2):136-46

24. Yousef AM, Al-Bakri AG, Bustanji Y, Wazaify M. Self-medication patterns in Amman, Jordan. Pharm World Sci. 2008;30:24-30. DOI:10.1007/ s11096-007-9135-x

25. Azami-Aghdash S, Mohseni M, Etemadi M, Royani S, Moosavi A, Nakhaee M. Prevalence and cause of self-medication in Iran: a systematic review and meta-analysis article. Iran J Public Health. 2015;44(12):1580-93

26. Ahmad A, Patel I, Mohanta GP, Balkrishnan R. 
Evaluation of self medication practices in rural area of town Sahaswan at northern India. Ann Med Health Sci Res. 2014; 4(Suppl 2): S73-S78. DOI: 10.4103/2141-9248.138012

27. Faria P, Freire T, Cordeiro K, Caetano P, Tolentino M, Gomes M. Prevalence and associated factors of self-medication in adults living in the Federal District, Brazil: a cross-sectional, population-based study. Epidemiol Serv Saude. 2017;26(2). DOI: 10.5123/S1679-49742017000200009

28. Shehnaz SI, Agarwal AK, Khan N. A systematic review of self-medication practices among adolescents. J Adolesc Health. 2014;55(4):467-83. DOI: 10.1016/j.jadohealth.2014.07.001
29. Machado-Alba J, Echeverri-Cataño E, LondoñoBuiles M, Moreno-Guitérrez P, Ochoa-Orozco S, Ruiz-Villa J. Social, cultural and economic factors associated with self-medication. Biomédica. 2014;34:580-8. DOI: http://dx.doi.org/10.7705/ biomedica.v34i4.2229

30. Pillaca-Medina ML, Carrión-Domínguez K. Automedicación en personas adultas que acuden a boticas del distrito Jesús Nazareno, Ayacucho 2015. An Fac med. 2016;77(4):387-92. DOI: http:// dx.doi.org/10.15381/anales.v77i4.12652

31. Carrasco-Garrido P, Jiménez-Garcia R, Barrera VH, Gil de Miguel A. Predictive factors of self-medicated drug use among the spanish adult population. Pharmacoepidemiol Drug Saf. 2008;17(2):193-9. DOI: 10.1002/pds.1455

32. Gómez LM, Galar M, Téllez AM, Carmona FA, Amaya A. Estudio de automedicación en una farmacia comunitaria de la ciudad de Toluca. Rev Mex Cienc Farm. 2009;40(1):5-11

33. Jerez-Roig J, Medeiros LF, Silva VA, Bezerra CL, Cavalcante LA, Piuvezam G, et al. Prevalence of self-medication and associated factors in an elderly population: a systematic review. Drugs Aging. 2014;31(12):883-96. DOI: 10.1007/s40266014-0217-x 